\title{
Innovation in IPE: let the students teach each other
}

Authors: Jonathan Berry and Anne O'Brien, School of Pharmacy and School of Health and Rehabilitation, Keele University

Background: Interprofessional education (IPE) has long been mooted as a method to improve teamwork, collaboration and clinical outcomes in healthcare (Barr et al 2011). Poorly designed IPE has the potential to reinforce stereotypes and power relationships between physicians and other healthcare professionals (Kuper and Whitehead 2012, Reeves and Van Schalk 2012). Students can become more sceptical of IPE the further they progress with their undergraduate studies (Pollard et al 2005). We aimed to design a teaching experience that would negate this scepticism.

Method: A bespoke interactive workshop was designed for final year pharmacy and physiotherapy students. Aims of the session were:

- To explore the differing approaches used by pharmacists and physiotherapists whilst dealing with clinical problems

- To share knowledge of the evidence base supporting the therapeutic management of patients

- To teach the other profession the key skills and concepts of their own therapeutic approach

- Discuss when it would be appropriate to refer a patient to a pharmacy/physiotherapy colleague

The session was evaluated with a bespoke pre and post workshop questionnaire.

Results: Since AY 2010/11, 516 students have undertaken the workshop. Prior to the session $59 \%$ of students stated that their previous IPE experience was not beneficial to their clinical practice, correlating with the findings of Pollard et al (2005). Student evaluation of this intervention has been consistently good; $92 \%$ of students this year stated that this IPE session had been extremely/ fairly useful; $85 \%$ stated the session would positively impact upon future clinical practice. In pre-session evaluation $41 \%$ of students were confident that they understood the role of the other profession; this improved to $98 \%$ after the workshop.

Discussion: Four successful iterations of this workshop have received positive student feedback. Students' confidence in their own clinical skills is improved by be given an opportunity to teach other professionals the skills they have learned. The design of any IPE session must take into account the previous experience and skills of students in order for it to be perceived as beneficial.

\section{References}

Barr H, Helme M, D'Avray L (2011). Developing interprofessional education in health and social care courses in the United Kingdom: A progress report. Occasional Paper 12, Health Sciences and Practice Subject Centre, the Higher education Academy.

Kuper A, Whitehead C (2012) The paradox of interprofessional education: IPE as a mechanism for maintaining physician power? Journal of Interporfessional Care 26; 347-349

Pollard K, Miers ME, Gilchrist M (2005) Second year scepticism: Pre-qualifying health and social care students' midpoint self-assessment, attitudes and perceptions concerning interprofessional learning and working. Journal of interprofessional care 19(3): 251-268

Reeves S and van Schalk S (2012) Simulation: A panacea for interprofessional learning? Journal of interprofessional care 26; 167-169. 